\title{
Correction to: Nitric Oxide Participates in the Brain Ischemic Tolerance Induced by Intermittent Hypobaric Hypoxia in the Hippocampal CA1 Subfield in Rats
}

\author{
Ya-Jie Huang ${ }^{1} \cdot$ Yu-Jia Yuan ${ }^{1} \cdot$ Yi-Xian Liu ${ }^{2} \cdot$ Meng-Yue Zhang $^{1}$ - Jing-Ge Zhang ${ }^{3}$ - Tian-Ci Wang ${ }^{1} \cdot$ Li-Nan Zhang $^{3}$. \\ $\mathrm{Yu}-\mathrm{Yan} \mathrm{Hu}^{3} \cdot \mathrm{Li} \mathrm{Li}^{4} \cdot$ Xiao-Hui Xian ${ }^{3} \cdot \mathrm{Jie} \mathrm{Qi}^{3} \cdot$ Min Zhang ${ }^{3,5}$
}

Published online: 31 August 2018

(c) Springer Science+Business Media, LLC, part of Springer Nature 2018

\section{Correction to: Neurochemical Research (2018) 43:1779-1790 \\ https://doi.org/10.1007/s11064-018-2593-9}

The order of corresponding author was inadvertently published. Hence, the first and the second corresponding authors should be Min Zhang (hebmuzhangmin@163.com) and Jing-Ge Zhang (zhangjg001@163.com).

The original article can be found online at https://doi.org/10.1007/ s11064-018-2593-9.

Jing-Ge Zhang

zhangjg001@163.com

$\triangle$ Min Zhang

hebmuzhangmin@163.com

1 Undergraduate of Clinical Medicine, Hebei Medical University, Shijiazhuang 050017, People's Republic of China

2 Department of Physiology, Hebei Medical University, Shijiazhuang 050017, People's Republic of China

3 Department of Pathophysiology, Hebei Medical University, No. 361 Zhongshan East Road, Shijiazhuang 050017, Hebei, People's Republic of China

4 Department of Science and Technology, The Second Hospital of Hebei Medical University, 215 Heping West Road, Shijiazhuang 050000, People's Republic of China

5 Aging and Cognition Neuroscience Laboratory of Hebei Province, Shijiazhuang 050017, People's Republic of China 